\title{
Determination of Insulin Resistance and Beta-Cell Function Using Homeostatic Model Assessment in Type 2 Diabetic Patients at Diagnosis
}

\author{
Prashant Basukala1*, Bharat Jha', Binod K. Yadav' and Pradeep Krishna Shrestha ${ }^{\mathbf{2}}$
}

${ }^{1}$ Department of Biochemistry, Institute of Medicine, Maharajgunj, Kathmandu, Nepal

${ }^{2}$ Department of Internal Medicine, Institute of Medicine, Maharajgunj, Kathmandu, Nepal

\begin{abstract}
Objective: Despite the increasing incidence of type 2 diabetes mellitus (T2DM) in South Asian countries, there have been no studies related to insulin resistance (IR) and beta-cell function (BCF) in Nepal. Measurement of both of these parameters at T2DM diagnosis can be a potential tool in evaluation, risk stratification and monitoring treatment. In this study, we used C-peptide modified homeostatic model assessment (both HOMA1 and HOMA2) and correlated the obtained IR with cardiovascular risk factors. We also intended to find out whether reduced insulin sensitivity or beta-cell failure predominates in new T2DM cases. Also there has been some dilemma in using either body mass index (BMI) or waist-hip ratio (WHR) as a better predictor of IR in our population. Lipoprotein ratios $\mathrm{TC} /$ HDL and TG/HDL also needed evaluation in this regard.

Results: Participants were sixty newly diagnosed T2DM patients visiting Tribhuvan University Teaching Hospital (TUTH), Nepal. The mean IR and beta-cell function were HOMA1IR=4.91 \pm 1.62 ; HOMA2IR=2.61 \pm 1.06 and HOMA1\%B=40.28 $\pm 23.64 ; \mathrm{HOMA} 2 \% \mathrm{~B}=47.10 \pm 24.67$ respectively. Both HOMA1 and HOMA2 showed greater reduction in insulin sensitivity than beta-cell function at diagnosis. ROC curves analysis showed WHR and TC/HDL ratio as better predictors of IR.
\end{abstract}

Keywords: Beta cell function; C-peptide; Cardiovascular risk factors; Diabetes mellitus; Insulin resistance; HOMA

\section{Abbreviations:}

\%B: Beta-Cell Function; \%S: Insulin Sensitivity; AUC: Area Under the Receiver Operating Characteristic Curve; BCF: Beta-Cell Function; BMI: Body Mass Index; DECODA: Diabetes Epidemiology Collaborative Analysis of Diagnostic Criteria in Asia; DECODE: Diabetes Epidemiology Collaborative Analysis of Diagnostic Criteria in Europe; FPG: Fasting Plasma Glucose; FBG: Fasting Blood Glucose; FPI: Fasting Plasma Insulin; HDL-C: High-Density Lipoprotein-Cholesterol; HOMA: Homeostasis Model Assessment; IR: Insulin Resistance; ISG: Insulin Sensitivity Group; LDL-C: Low-Density Lipoprotein Cholesterol; ROC: Receiver Operating Characteristic; T2DM: Type 2 Diabetes Mellitus; TUTH: Tribhuvan University Teaching Hospital; WHR: Waist-Hip Ratio

\section{Introduction}

Insulin resistance (IR) and beta-cell dysfunction are characteristic features of type 2 diabetes mellitus (T2DM). Insulin resistance is characterized by decrease in insulin mediated glucose disposal in insulin-sensitive tissue and increased hepatic glucose production [1] whereas beta-cell dysfunction occurs when beta-cells are unable to compensate for the insulin resistance [2]. Measurement of both of these parameters at diagnosis of T2DM can be a potential tool in evaluation, risk stratification and monitoring treatment of DM.

Hyperinsulinemic-euglycemic clamp technique is the gold standard method for determining IR. However it is cumbersome to perform, labor-intensive and costly [3]. Homeostatic Model Assessment (HOMA) test introduced in 1985 by David Matthews et al. is a simple test that mathematically models the fasting plasma glucose and insulin concentrations and gives an estimate of individual's degree of insulin sensitivity (HOMA \%S) and level of beta-cell function (HOMA \%B). HOMA-IR is the reciprocal of HOMA \%S [4]. In 1998, Jonathan Levy et al published an updated HOMA model (HOMA2) which along with the steady state glucose and insulin ratio also considers variations in hepatic and peripheral glucose resistance i.e., the reduction in the suppression of hepatic glucose output (by hyperglycemia), increases in the insulin secretion curve for plasma glucose concentrations above $10 \mathrm{mmol} / \mathrm{L}$ and the effects of circulating proinsulin [5]. Later, in 2004, a computer model called the HOMA Calculator was released which could provide quick and easy access to the HOMA2 model [6].

However, these original models which use insulin have some limitations. First, the insulin secretion is pulsatile, which limits the use of a single sample for insulin determination. Instead, we had to use the mean of at least three samples taken at 5-min intervals to compute HOMA for more reliable results. Also careful phlebotomy is essential to prevent hemolysis as far as possible as hemolysis results in degradation of insulin. Moreover, nearly $50 \%$ of insulin secreted by beta-cells are extracted by the liver causing a large insulin inter assay variation [7].

In China, $\mathrm{Li}$ et al. [8] found a modified homeostasis model assessment (modified HOMA) using C-peptide to replace insulin in HOMA to evaluate both IR and islet cell function in normal and diabetic patients. This C-peptide modified HOMA seems more appropriate as a measure of insulin secretion as the equimolarly secreted C-peptide is not extracted by the liver and other organs and also, the half-life of C-peptide in blood is quite longer than that of insulin (10-30 min vs. 4 min) making

${ }^{*}$ Corresponding author: Prashant Basukala, Department of Biochemistry, Institute of Medicine, Maharajgunj, Kathmandu, Nepal, Tel: +9779849177116; E-mail: basukalaprashant@gmail.com

Received March 06, 2018; Accepted March 18, 2018; Published March 22, 2018

Citation: Basukala P, Jha B, Yadav BK, Shrestha PK (2018) Determination of Insulin Resistance and Beta-Cell Function Using Homeostatic Model Assessment in Type 2 Diabetic Patients at Diagnosis. J Diabetes Metab 9: 790 doi: 10.4172/2155-6156.1000790

Copyright: () 2018 Basukala P, et al. This is an open-access article distributed under the terms of the Creative Commons Attribution License, which permits unrestricted use, distribution, and reproduction in any medium, provided the original author and source are credited. 
the measurement of C-peptide a more reliable representative of beta-cell insulin secretion compared to the measurement of insulin itself [2].

Insulin resistance has been well known as the major player in progression from metabolic syndrome to overt diabetes mellitus. IR as assessed by HOMA indices has also been correlated with intima-media thickness of the carotid artery [9] which is a well-accepted marker of atherosclerosis. Also, the lipid markers like plasma triglyceride (TG), high-density lipoprotein cholesterol (HDL-C), and total cholesterol (TC) have been reported to be independently associated with insulin resistance, and are independent predictors of cardiovascular disease (CVD) [10]. Lipoprotein ratios such as TC/HDL-C and TG/HDL-C ratios which are derived from these lipid markers, have been shown by several epidemiological studies as better predictors of CVD than any single lipid marker [11-13]. One of the major advantages of these ratios is that they can be promptly calculated by the physician and are easier to comprehend at the clinical level.

Studies have also shown that, it takes 5-10 years before actual symptoms of diabetes start to appear due to defect in beta-cell function (BCF) and insulin resistance. Under such scenario, early detection of insulin resistance and beta-cell function could be of potential benefit for applying preventive measures to attenuate the progression to overt diabetes. Various dynamic and steady state tests are available for this but each has their own pros and cons. There is a paucity of nationwide data regarding this. So this study has been designed with the following objectives:

-To determine insulin resistance and beta-cell function using modified HOMA in newly diagnosed type 2 diabetic patients.

-To know whether reduced insulin sensitivity or beta-cell failure predominates in newly diagnosed type 2 diabetic patients.

- To correlate insulin resistance with BMI and waist hip ratio (WHR).

-To correlate insulin resistance and beta-cell function with other cardiovascular risk factors such as hypertension, family history of DM, dietary habit, stress, exercise, alcohol intake, smoking, tobacco intake, and lipid profile.

We also investigated the reliability of TC/HDL-C and TG/HDL-C ratios as surrogate marker of insulin resistance.

\section{Materials and Methods}

\section{Place and duration of study}

This study was conducted at Department of Endocrinology and Biochemistry Laboratory of Tribhuvan University Teaching Hospital (TUTH), a tertiary care centre in Kathmandu, Nepal from February 2016 to January 2017.

\section{Study population, inclusion and exclusion criteria}

Sixty newly diagnosed T2DM patients who provided written consent were enrolled in this study. Exclusion criteria included patients with evident major diabetic complications, such as heart disease and diabetic nephropathy, patients suffering from chronic illness, chronic liver and/ or renal diseases, patients already receiving lipid-lowering medications and/or oral hypoglycemic drugs, and pregnant women. Participants were interviewed by the principal investigator by asking the questions included in questionnaire (self-designed semi-structured proforma). Thirty age and sex matched non-diabetic apparently healthy individuals were recruited as control.

\section{Anthropometric measurements}

Weight was taken using a platform weighing scale. Standing height measurement was done with participants in bare foot, eyes looking ahead. The waist circumference was measured at the midpoint between the lowest rib and iliac crest and at the end of expiration. The hip circumference was taken at the widest area of the hips at the greatest protuberance of the buttocks. BMI was calculated by weight in kilograms divided by square of height in meters. WHR was calculated by simply dividing the waist measurement by the hip measurement. Blood pressure (BP) measurement was done using a recently calibrated aneroid sphygmomanometer with an adequate cuff size after participant has rested for at least 5 minutes.

\section{Collection and processing of the sample}

Five millilitre of blood was drawn after an overnight fast (8 -12 hours) by venous puncture method. Serum samples were separated, within half an hour, by centrifugation at $1500-3000 \mathrm{rpm}$ for $5 \mathrm{~min}$. Routine investigation were done on the same day of sample collection, which included blood glucose, creatinine, SGPT, TC, HDL-cholesterol and TG, which were measured in fully-automated biochemistry analyser, BT 3000 , Italy. An aliquot of each sample was then stored at $-20^{\circ} \mathrm{C}$ for the test of C-peptide.

Laboratory standard operating procedures were maintained for all laboratory analysis. Internal quality control sera, both normal and pathological, were also run for each lot, for the validation of the results.

Fasting serum glucose was measured by glucose oxidase method, as described by Trinder, using commercial kit Biolabo Reagents, France. Serum creatinine was measured by modified Jaffe reaction, Biolabo Reagents, France. SGPT was measured by IFCC recommended kinetic method. Total cholesterol (TC) was estimated by CHOD/PAP method, Human, Germany. Triglyceride (TG) was measured by GPO/PAP method, Human, Germany. HDL-C was measured by PEG/CHOD-PAP method, Human, Germany. LDL-C was calculated using the Friedewald's formula:- LDL-C $(\mathrm{mmol} / \mathrm{L})=\mathrm{TC}(\mathrm{mmol} / \mathrm{L})-\mathrm{HDL}-\mathrm{C}(\mathrm{mmol} / \mathrm{L})-\mathrm{TG}$ $(\mathrm{mmol} / \mathrm{L}) / 2.2$. When TG concentration exceeded $4 \mathrm{mmol} / \mathrm{l}$, LDL-C was estimated by direct homogenous method, Biolabo Reagents, France. VLDL-C $(\mathrm{mmol} / \mathrm{L})$ was calculated as TG $(\mathrm{mmol} / \mathrm{L}) / 2.2$.

C-peptide was measured using a solid phase enzyme-linked immunosorbent assay (ELISA) kit, DRG, Italy. The HOMA model was used to calculate insulin resistance, insulin sensitivity, and beta-cell function using the C-peptide modified formulae given by Li et al. [8]

The modified HOMA formulas were:-

HOMA1-IR $=1.5+$ FPG $\times$ FCP $/ 2800$

HOMA1 \%B $($ Normal $)=0.27 \times$ FCP $/($ FPG-3.5 $)+50$

HOMA1 \%B $(\mathrm{DM})=0.27 \times \mathrm{FCP} /(\mathrm{FPG}-3.5)$

where,

$\mathrm{FPG}=$ Fasting plasma glucose $(\mathrm{mmol} / \mathrm{L})$

FCP = Fasting C-peptide (pmol/L)

HOMA1 \%S was calculated by reciprocal of HOMA1-IR multiplied by $100 \%$. Patients with HOMA1-IR $>2.5$ were defined as having insulin resistance whereas those with HOMA1-IR $\leq 2.5$ were defined as insulin sensitivity group $[13,14]$. IR and BCF values were also calculated using the HOMA2 calculator software. HOMA2 calculator was downloaded from university of oxford. http://www.dtu.ox.ac.uk/. Patients with 
HOMA2-IR > 1.8 were defined as having insulin resistance whereas those with HOMA2-IR $\leq 1.8$ were defined as insulin sensitivity group (ISG) [15]. The calculated HOMA1-IR and HOMA2-IR were compared in individual patients. HOMA2 being more accurate representation of glucose homeostasis than HOMA1 [7], was used to compare various physical, socio-demographic, laboratory and clinical parameters between the insulin resistant and ISG of the diabetic cases in this study. Diabetic patients were also separated into two groups on the basis of presentation with osmotic symptoms (polyuria, polydipsia, polyphagia, and dehydration) and without osmotic symptoms and these two groups were compared for biochemical parameters.

\section{Data processing and analysis}

The data were entered in Microsoft Excel program (Microsoft Office 2010). Statistical analyses were done by SPSS 23.0 version (Statistical Package for Social Science for Window version; SPSS, Inc., Chicago, IL). Mean comparison was done by t-test. Chi square test was used for comparison of dichotomous variables. Pearson correlation was used to evaluate the correlation between cardiovascular risk factors and insulin resistance. P value $\leq 0.05$ was considered to be statistically significant.

Evaluation of serum lipoprotein ratios was done by constructing receiver operating characteristic (ROC) curve to examine the predictive value of TC/HDL-C, and TG/ HDL-C ratios for insulin resistance (HOMA2-IR > 1.8). Values for the area under the ROC curve of 0.5 , $\geq 0.7$ but $<0.8, \geq 0.8$ but $<0.9$, and $\geq 0.9$ were taken as suggestive of reflecting the following levels of discrimination: none, acceptable, excellent, and outstanding [16].

\section{Results}

The mean age of type 2 diabetic patients at diagnosis was $46.0 \pm$ 7.8 years $(n=60)$. The age ranged from 33 years to 66 years. This study population included 36 males and 24 females. The mean age for male patients was $44.9 \pm 8.4$ years and for female patients was $47.7 \pm 6.6$ years. The mean age of control individuals was $44.5 \pm 8.4$ years $(n=30)$. Basic characteristics of case and control are depicted in Table 1. Fasting blood glucose, fasting $\mathrm{C}$-peptide, insulin resistance as assessed by HOMA1 and HOMA2, serum TG, TC/HDL-C ratio, and TG/HDL-C ratio were significantly higher in cases than controls; whereas insulin sensitivity and beta-cell function as assessed by HOMA1 and HOMA2 were significantly lower in cases than controls. Both HOMA1 and HOMA2 showed greater reduction in insulin sensitivity (i.e. higher insulin resistance) than reduction in beta-cell function in diabetic cases (Table 1). This indicates that the development of insulin resistance proceeds over deterioration of beta-cell secretory capacity in newly diagnosed type 2 diabetic patients in our settings.

Among 60 diabetic cases, HOMA1 identified 56 patients as insulin resistant and only 4 patients as insulin sensitivity group with the cut off of 2.5 whereas HOMA2 identified 43patients as insulin resistant and 17 patients as insulin sensitivity group with a cut off of 1.8. Concordant results were obtained for 47 patients whereas 13 HOMA1 insulin resistant patients were deemed under insulin sensitivity group by HOMA2 (Table 2). HOMA2-IR gave comparatively lower indexes than HOMA1-IR. Scatter plot relationship between HOMA1 and HOMA2 is shown in Figure 1. Using HOMA2, significant mean differences in insulin resistant and insulin sensitive diabetic groups were found for LDL cholesterol and waist hip ratio (Table 3) and for age group, presence of osmotic symptoms, family history of diabetes, adequacy of vegetables intake, and stressful lifestyle (Table 4). Similarly, between patients with osmotic symptoms and without osmotic symptoms, significant difference in means were obtained for FBG, FCP, HOMA1-IR, HOMA1 $\%$ S, HOMA $1 \%$ B, HOMA2-IR, HOMA $2 \% S$ and HOMA2 \%B (Table 5). Higher insulin resistance was seen in patients presented with osmotic symptoms than those without osmotic symptoms (Figure 2). Significant positive correlations were obtained in both HOMA1-IR and HOMA2IR for LDL cholesterol, waist hip ratio, blood pressure, family history of diabetes and stress (Table 6). Oil intake correlated significantly with HOMA1-IR and tobacco intake correlated significantly with HOMA2IR. Similarly, significant negative correlations were obtained in both HOMA1 \%B and HOMA2 \%B for LDL cholesterol, family history of diabetes, oil intake, salt intake and stressful lifestyle (Table 7). Exercise had significant positive correlation with both HOMA1 \%B and HOMA2 $\% \mathrm{~B}$ and alcohol intake had significant negative correlation with HOMA2 $\% \mathrm{~B}$.

Finally, ROC curves were drawn for both BMI and WHR for prediction of insulin resistance (Figure 3). HOMA2-IR was used as gold standard ( $>1.8$ was taken as positive for insulin resistance). The AUC for prediction of insulin resistance for WHR was significantly higher than for BMI (Table 7). Lipoprotein ratios: TC/HDL-C and TG/HDL-C were also analyzed using ROC curves for prediction of insulin resistance (Figure $4 \mathrm{a}$ and $4 \mathrm{~b}$ ). TC/HDL-C ratio was found to have acceptable

\begin{tabular}{|c|c|c|c|c|c|}
\hline & \multicolumn{2}{|c|}{ Case Diabetic patients) $n=60$} & \multicolumn{2}{|c|}{ Control(Non-diabetic; apparently healthy individuals) $n=30$} & \multirow{2}{*}{$p$-value } \\
\hline & Mean & SD & Mean & SD & \\
\hline Age (years) & 46.0 & 7.8 & 44.5 & 8.4 & 0.395 \\
\hline Fasting Blood Glucose (mmol/l) & 10.68 & 3.46 & 4.90 & 0.52 & $<0.001^{*}$ \\
\hline Fasting C-peptide (pmol/l) & 875.41 & 294.53 & 534.25 & 143.22 & $<0.001^{*}$ \\
\hline HOMA1-IR & 4.91 & 1.62 & 2.43 & 0.26 & $<0.001^{*}$ \\
\hline HOMA1 \%S & 22.84 & 8.25 & 41.57 & 4.76 & $<0.001^{*}$ \\
\hline HOMA1 \%B & 40.28 & 23.64 & 168.60 & 53.69 & $<0.001^{*}$ \\
\hline HOMA2-IR & 2.61 & 1.06 & 1.16 & 0.31 & $<0.001^{*}$ \\
\hline HOMA2 \%S & 46.34 & 22.81 & 93.91 & 32.61 & $<0.001^{*}$ \\
\hline HOMA2 \%B & 47.10 & 24.67 & 113.10 & 30.56 & $<0.001^{*}$ \\
\hline Total Cholesterol (mmol/l) & 4.55 & 0.96 & 4.21 & 0.68 & 0.086 \\
\hline Triglyceride $(\mathrm{mmol} / \mathrm{l})$ & 2.33 & 1.43 & 1.18 & 0.56 & $<0.001^{*}$ \\
\hline HDL Cholesterol (mmol/l) & 1.00 & 0.22 & 1.06 & 0.16 & 0.249 \\
\hline LDL Cholesterol (mmol/l) & 2.68 & 0.87 & 2.59 & 0.50 & 0.526 \\
\hline TC/HDL-C ratio & 4.60 & 0.93 & 4.00 & 0.49 & $<0.001^{*}$ \\
\hline TG/HDL-C ratio & 2.37 & 1.61 & 1.15 & 0.59 & $<0.001^{*}$ \\
\hline
\end{tabular}

*Statistically significant at $\mathrm{p}<0.05$; Independent Sample $t$ test

Table 1: Comparison of different physical and laboratory parameters between case (diabetic patients) and control (apparently healthy individuals). 
Citation: Basukala P, Jha B, Yadav BK, Shrestha PK (2018) Determination of Insulin Resistance and Beta-Cell Function Using Homeostatic Model Assessment in Type 2 Diabetic Patients at Diagnosis. J Diabetes Metab 9: 790. doi: 10.4172/2155-6156.1000790

Page 4 of 11

\begin{tabular}{|c|c|c|c|c|c|}
\hline Patient S. No. & HOMA-IR & Categorization based on cut-off of 2.5 & HOMA2-IR & Categorization based on cut-off of 1.8 & $\begin{array}{c}\text { Concordance between HOMA-IR and } \\
\text { HOMA2-IR }\end{array}$ \\
\hline 1 & 6.22 & IR & 4.59 & IR & Yes \\
\hline 2 & 5.48 & IR & 2.80 & IR & Yes \\
\hline 3 & 5.94 & IR & 3.18 & IR & Yes \\
\hline 4 & 4.66 & IR & 2.77 & IR & Yes \\
\hline 5 & 5.89 & IR & 3.00 & IR & Yes \\
\hline 6 & 7.68 & IR & 4.03 & IR & Yes \\
\hline 7 & 6.34 & IR & 3.40 & IR & Yes \\
\hline 8 & 5.49 & IR & 3.82 & IR & Yes \\
\hline 9 & 4.36 & IR & 2.69 & IR & Yes \\
\hline 10 & 3.82 & IR & 2.22 & IR & Yes \\
\hline 11 & 3.75 & IR & 1.74 & ISG & No \\
\hline 12 & 3.23 & IR & 1.50 & ISG & No \\
\hline 13 & 2.95 & IR & 1.29 & ISG & No \\
\hline 14 & 5.59 & IR & 2.77 & IR & Yes \\
\hline 15 & 5.13 & IR & 2.88 & IR & Yes \\
\hline 16 & 3.42 & IR & 1.69 & ISG & No \\
\hline 17 & 6.02 & IR & 3.00 & IR & Yes \\
\hline 18 & 3.93 & IR & 1.73 & ISG & No \\
\hline 19 & 3.40 & IR & 1.63 & ISG & No \\
\hline 20 & 4.74 & IR & 2.20 & IR & Yes \\
\hline 21 & 9.09 & IR & 5.68 & IR & Yes \\
\hline 22 & 4.31 & IR & 2.03 & IR & Yes \\
\hline 23 & 2.49 & ISG & 1.00 & ISG & Yes \\
\hline 24 & 3.20 & IR & 1.53 & ISG & No \\
\hline 25 & 2.59 & IR & 1.03 & ISG & No \\
\hline 26 & 6.70 & IR & 3.75 & IR & Yes \\
\hline 27 & 2.44 & ISG & .92 & ISG & Yes \\
\hline 28 & 3.49 & IR & 2.21 & IR & Yes \\
\hline 29 & 9.39 & IR & 5.29 & IR & Yes \\
\hline 30 & 5.60 & IR & 2.72 & IR & Yes \\
\hline 31 & 2.30 & ISG & 0.93 & ISG & Yes \\
\hline 32 & 4.15 & IR & 1.93 & IR & Yes \\
\hline 33 & 6.47 & IR & 3.91 & IR & Yes \\
\hline 34 & 4.33 & IR & 2.27 & IR & Yes \\
\hline 35 & 6.36 & IR & 3.36 & IR & Yes \\
\hline 36 & 3.26 & IR & 1.46 & ISG & No \\
\hline 37 & 4.98 & IR & 2.48 & IR & Yes \\
\hline 38 & 6.48 & IR & 3.30 & IR & Yes \\
\hline 39 & 5.01 & IR & 2.73 & IR & Yes \\
\hline 40 & 3.75 & IR & 2.39 & IR & Yes \\
\hline 41 & 6.10 & IR & 3.70 & IR & Yes \\
\hline 42 & 5.07 & IR & 2.83 & IR & Yes \\
\hline 43 & 5.14 & IR & 3.22 & IR & Yes \\
\hline 44 & 3.28 & IR & 1.52 & ISG & No \\
\hline 45 & 6.68 & IR & 3.44 & $\mathrm{IR}$ & Yes \\
\hline 46 & 5.36 & IR & 2.53 & IR & Yes \\
\hline 47 & 7.64 & IR & 4.02 & IR & Yes \\
\hline 48 & 4.76 & IR & 2.76 & IR & Yes \\
\hline 49 & 5.58 & IR & 3.76 & IR & Yes \\
\hline 50 & 4.84 & IR & 2.84 & IR & Yes \\
\hline 51 & 2.29 & ISG & 1.02 & ISG & Yes \\
\hline 52 & 3.73 & IR & 1.59 & ISG & No \\
\hline 53 & 4.70 & IR & 2.13 & IR & Yes \\
\hline 54 & 2.84 & IR & 1.29 & ISG & No \\
\hline 55 & 5.31 & IR & 2.65 & IR & Yes \\
\hline 56 & 2.77 & IR & 1.22 & ISG & No \\
\hline 57 & 4.82 & IR & 2.51 & IR & Yes \\
\hline 58 & 5.58 & IR & 2.76 & IR & Yes \\
\hline 59 & 6.68 & IR & 3.55 & IR & Yes \\
\hline
\end{tabular}


Citation: Basukala P, Jha B, Yadav BK, Shrestha PK (2018) Determination of Insulin Resistance and Beta-Cell Function Using Homeostatic Model Assessment in Type 2 Diabetic Patients at Diagnosis. J Diabetes Metab 9: 790. doi: 10.4172/2155-6156.1000790

Page 5 of 11

\begin{tabular}{|c|c|c|c|c|}
\hline 60 & 6.92 & IR & 3.57 & IR \\
\hline \multirow{2}{*}{ Total } & & IR $=56(93.3 \%)$ & IR $=43(71.7 \%)$ & Yes $=47(78.3 \%)$ \\
\cline { 2 - 5 } & & ISG $=4(6.7 \%)$ & ISG $=17(28.3 \%)$ \\
\hline
\end{tabular}

IR: Insulin Resistant

ISG: Insulin Sensitivity Group

Table 2: Comparison of HOMA1-IR and HOMA2-IR in categorization of individual patients with respective cut-off values and concordance between them.

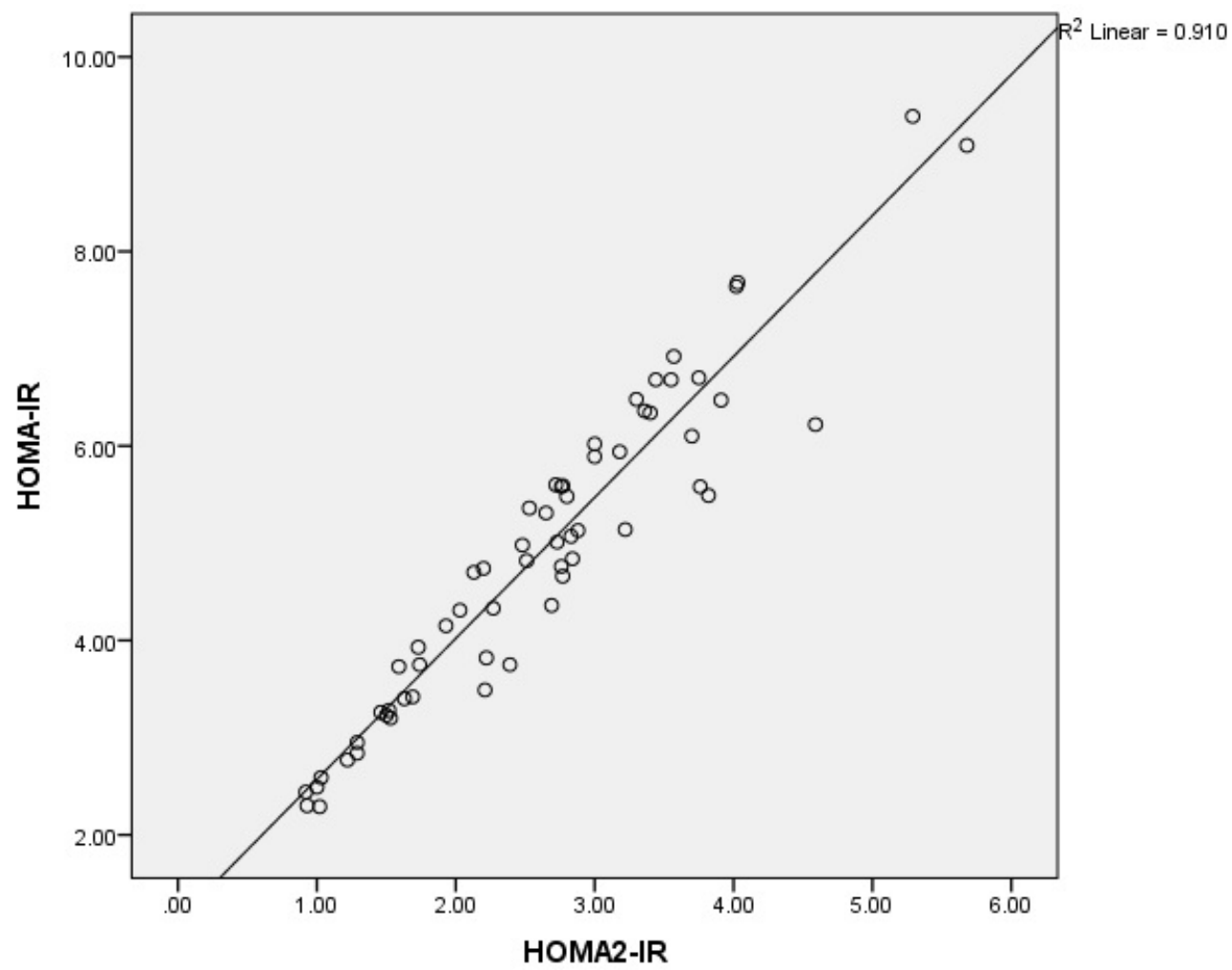

Figure 1: Scatterplot relationship between HOMA1-IR and HOMA2-IR.

\begin{tabular}{|c|c|c|c|c|c|}
\hline & \multicolumn{2}{|c|}{ HOMA2-IR $\leq 1.8(n=17)$} & \multicolumn{2}{|c|}{ HOMA2-IR >1.8 (n=43) } & \multirow[b]{2}{*}{ p-value } \\
\hline & Mean & \pm SD & Mean & \pm SD & \\
\hline Age (years) & 44.9 & 9.0 & 46.5 & 7.3 & 0.501 \\
\hline Fasting Plasma Glucose (mmol/l) & 8.04 & 1.75 & 11.73 & 3.42 & $<0.001^{*}$ \\
\hline Fasting C-peptide (pmol/l) & 529.2 & 87.6 & 1012.3 & 226.3 & $<0.001^{*}$ \\
\hline HOMA-IR & 3.05 & 0.52 & 5.6430 & 1.27 & $<0.001^{*}$ \\
\hline HOMA \%S & 33.71 & 5.91 & 18.55 & 3.95 & $<0.001^{*}$ \\
\hline HOMA \%B & 36.89 & 19.82 & 41.62 & 25.08 & 0.490 \\
\hline HOMA2-IR & 1.36 & 0.29 & 3.11 & 0.82 & $<0.001^{*}$ \\
\hline HOMA2 \%S & 77.27 & 18.22 & 34.12 & 8.00 & $<0.001^{*}$ \\
\hline HOMA2 \%B & 47.91 & 19.71 & 46.79 & 26.58 & 0.876 \\
\hline Total Cholesterol (mmol/l) & 4.33 & 0.99 & 4.64 & 0.95 & 0.267 \\
\hline Triglyceride $(\mathrm{mmol} / \mathrm{l})$ & 2.26 & 1.05 & 2.36 & 1.56 & 0.802 \\
\hline HDL Cholesterol (mmol/l) & 1.02 & 0.23 & 1.00 & 0.21 & 0.777 \\
\hline LDL Cholesterol (mmol/l) & 2.33 & 0.81 & 2.82 & 0.85 & $0.046^{*}$ \\
\hline TC/HDL-C ratio & 4.32 & 0.84 & 4.71 & 0.95 & 0.149 \\
\hline TG/HDL-C ratio & 2.19 & 0.83 & 2.44 & 1.83 & 0.484 \\
\hline Body Mass Index (BMI) & 27.49 & 3.75 & 28.75 & 3.39 & 0.214 \\
\hline Waist Circumference (cm) & 104.18 & 10.68 & 102.65 & 11.28 & 0.634 \\
\hline Waist Hip Ratio (WHR) & 1.00 & 0.04 & 1.04 & 0.05 & $0.014^{*}$ \\
\hline
\end{tabular}

*Statistically significant at $p<0.05$; Independent Sample $t$ test

Table 3: Comparing different physical and laboratory parameters between patients with HOMA2-IR $\leq 1.8$ and $>1.8$. 
Citation: Basukala P, Jha B, Yadav BK, Shrestha PK (2018) Determination of Insulin Resistance and Beta-Cell Function Using Homeostatic Model Assessment in Type 2 Diabetic Patients at Diagnosis. J Diabetes Metab 9: 790. doi: 10.4172/2155-6156.1000790

Page 6 of 11

\begin{tabular}{|c|c|c|c|c|c|c|c|}
\hline & & \multicolumn{2}{|c|}{$\begin{array}{l}\text { HOMA2-IR } \\
\leq 1.8(n=17)\end{array}$} & \multicolumn{2}{|c|}{$\begin{array}{l}\text { HOMA2-IR } \\
>1.8(n=43)\end{array}$} & \multirow[t]{2}{*}{ Total } & \multirow[t]{2}{*}{$p$-value } \\
\hline & & No. & $\%$ & No. & $\%$ & & \\
\hline \multirow{4}{*}{ Age Group } & $31-40$ & 7 & 36.8 & 12 & 63.2 & 19 & \multirow{4}{*}{$0.012^{*}$} \\
\hline & $41-50$ & 8 & 30.8 & 18 & 69.2 & 26 & \\
\hline & $51-60$ & 0 & 0 & 13 & 100 & 13 & \\
\hline & $>60$ & 2 & 100 & 0 & 0 & 2 & \\
\hline \multirow{2}{*}{ Gender } & Male & 12 & 33.3 & 24 & 66.7 & 36 & \multirow{2}{*}{0.293} \\
\hline & Female & 5 & 20.8 & 19 & 79.2 & 24 & \\
\hline \multirow{2}{*}{ Obesity $^{\mathrm{a}}$ (BMI) } & Non-obese & 6 & 46.2 & 7 & 53.8 & 13 & \multirow{2}{*}{0.107} \\
\hline & Obese & 11 & 23.4 & 36 & 76.6 & 47 & \\
\hline \multirow{2}{*}{$\begin{array}{c}\text { Obesity }^{\mathrm{b}} \\
\text { (Waist circumference) }\end{array}$} & Non-obese & 2 & 33.3 & 4 & 66.7 & 6 & \multirow{2}{*}{0.774} \\
\hline & Obese & 15 & 27.8 & 39 & 72.2 & 54 & \\
\hline \multirow{4}{*}{ Obesity (Waist Hip Ratio) } & $0.80-0.89$ & 0 & 0 & 0 & 0 & 0 & \multirow{4}{*}{0.079} \\
\hline & $0.90-0.99$ & 8 & 50 & 8 & 50 & 16 & \\
\hline & $1.00-1.09$ & 8 & 20 & 32 & 80 & 40 & \\
\hline & $1.10-1.19$ & 1 & 25 & 3 & 75 & 4 & \\
\hline \multirow{2}{*}{ Osmotic symptoms } & No & 15 & 71.4 & 6 & 28.6 & 21 & \multirow{2}{*}{$<0.001^{*}$} \\
\hline & Yes & 2 & 5.1 & 37 & 94.9 & 39 & \\
\hline \multirow{2}{*}{ Hypertension` (BP systolic) } & No & 17 & 32.1 & 36 & 67.9 & 53 & \multirow{2}{*}{0.077} \\
\hline & Yes & 0 & 0 & 7 & 100 & 7 & \\
\hline Hypertencinnd (RP diactolic) & No & 17 & 32.7 & 35 & 67.3 & 52 & \\
\hline Hypertension (BP diastolıc) & Yes & 0 & 0 & 8 & 100 & 8 & 0.056 \\
\hline Family History & No & 15 & 38.5 & 24 & 61.5 & 39 & $0018^{*}$ \\
\hline of DM & Yes & 2 & 9.5 & 19 & 90.5 & 21 & 0.018 \\
\hline Dietary Hahbit & Vegetarian & 2 & 66.7 & 1 & 33.3 & 3 & 0131 \\
\hline Dletaly navoli & Non-vegetarian & 15 & 26.3 & 42 & 73.7 & 57 & 0.101 \\
\hline & 2 times daily & 4 & 30.8 & 9 & 69.2 & 13 & \\
\hline & 3 times daily & 10 & 30.3 & 23 & 69.7 & 33 & 0.807 \\
\hline & 4 times daily & 3 & 21.4 & 11 & 78.6 & 14 & \\
\hline Evorcise & No & 16 & 29.6 & 38 & 70.4 & 54 & 0504 \\
\hline Exercise & Yes & 1 & 16.7 & 5 & 83.3 & 6 & 0.504 \\
\hline & Low & 2 & 9.5 & 19 & 90.5 & 21 & \\
\hline Vegetables intake ${ }^{e}$ & Normal & 15 & 39.5 & 23 & 60.5 & 38 & $0.041^{*}$ \\
\hline & High & 0 & 0 & 1 & 100 & 1 & \\
\hline & Low & 0 & 0 & 1 & 100 & 1 & \\
\hline Carbohydrates Intake & Normal & 5 & 29.4 & 12 & 70.6 & 17 & 0.816 \\
\hline & High & 12 & 28.6 & 30 & 71.4 & 42 & \\
\hline & Low & 11 & 32.4 & 23 & 67.6 & 34 & \\
\hline Fruits intake & Normal & 6 & 24 & 19 & 76 & 25 & 0.639 \\
\hline & High & 0 & 0 & 1 & 100 & 1 & \\
\hline & Low & 0 & 0 & 3 & 100 & 3 & \\
\hline Oil intake & Normal & 12 & 34.3 & 23 & 65.7 & 35 & 0.343 \\
\hline & High & 5 & 22.7 & 17 & 77.3 & 22 & \\
\hline & Low & 0 & 0 & 4 & 100 & 4 & \\
\hline Salt intake & Normal & 15 & 29.4 & 36 & 70.6 & 51 & 0.378 \\
\hline & High & 2 & 40 & 3 & 60 & 5 & \\
\hline Lifestvle & Non-Stressful & 17 & 43.6 & 22 & 56.4 & 39 & $<0001^{*}$ \\
\hline Lilestyle & Stressful & 0 & 0 & 21 & 100 & 21 & $<0.001$ \\
\hline Alcohol intake & No & 7 & 28 & 18 & 72 & 25 & 0061 \\
\hline Alconol intake & Yes & 10 & 28.6 & 25 & 71.4 & 35 & 0.961 \\
\hline Cmoking & No & 14 & 34.1 & 27 & 65.9 & 41 & 0112 \\
\hline SIMOKIng & Yes & 3 & 15.8 & 16 & 84.2 & 19 & 0.142 \\
\hline Toharco & No & 14 & 30.4 & 32 & 69.6 & 46 & 0513 \\
\hline IODacco & Yes & 3 & 21.4 & 11 & 78.6 & 14 & 0.513 \\
\hline
\end{tabular}

*Statistically significant at $\mathrm{p}<0.05$; Chi-square test

${ }^{a}$ Classification was based on two cut-off points of $<25$ for non-obese and $\geq 25$ for obese

${ }^{\mathrm{b}}$ Classification was based on two cut-off points for male $(\geq 90 \mathrm{~cm})$ and female $(\geq 80 \mathrm{~cm})$

${ }^{\circ}$ Classification was based on cut-off point of $140 \mathrm{mmHg}$

${ }^{\mathrm{d} C l a s s i f i c a t i o n ~ w a s ~ b a s e d ~ o n ~ c u t-o f f ~ p o i n t ~ o f ~} 90 \mathrm{mmHg}$

eClassification was based on asking the participant whether he/she prefers taking it in that amount compared to other family members, relatives, or friends.

Table 4: Relation between Insulin Resistance (HOMA2-IR) and Socio-demographic and clinical characteristics. 
Citation: Basukala P, Jha B, Yadav BK, Shrestha PK (2018) Determination of Insulin Resistance and Beta-Cell Function Using Homeostatic Model Assessment in Type 2 Diabetic Patients at Diagnosis. J Diabetes Metab 9: 790. doi: 10.4172/2155-6156.1000790

Page 7 of 11

\begin{tabular}{|c|c|c|c|c|c|}
\hline & \multicolumn{2}{|c|}{ Diabetic patients with osmotic symptoms $(n=39)$} & \multicolumn{2}{|c|}{ Diabetic patients without osmotic symptoms $(n=21)$} & \multirow[b]{2}{*}{$p$-value } \\
\hline & Mean & SD & Mean & SD & \\
\hline Age (years) & 46.8 & 7.9 & 44.6 & 7.5 & 0.307 \\
\hline Fasting Blood Glucose (mmol/l) & 12.29 & 3.11 & 7.69 & 1.59 & $<0.001^{*}$ \\
\hline Fasting C-peptide (pmol/l) & 1000.78 & 248.46 & 642.58 & 225.93 & $<0.001^{*}$ \\
\hline HOMA1-IR & 5.79 & 1.23 & 3.27 & 0.69 & $<0.001^{*}$ \\
\hline HOMA $1 \% S$ & 17.98 & 3.57 & 31.89 & 6.67 & $<0.001^{*}$ \\
\hline HOMA1 \%B & 35.90 & 19.84 & 48.41 & 28.17 & $0.081^{*}$ \\
\hline HOMA2-IR & 3.15 & 0.86 & 1.62 & 0.57 & $<0.001^{*}$ \\
\hline HOMA2 \%S & 34.04 & 9.25 & 69.19 & 22.99 & $<0.001^{*}$ \\
\hline HOMA2 \%B & 40.58 & 20.66 & 59.22 & 27.33 & $0.004^{*}$ \\
\hline
\end{tabular}

*Statistically significant at $\mathrm{p}<0.05$; Independent Sample $t$ test

Table 5: Comparison of different physical and laboratory parameters in diabetic patients with osmotic symptoms and without osmotic symptoms.

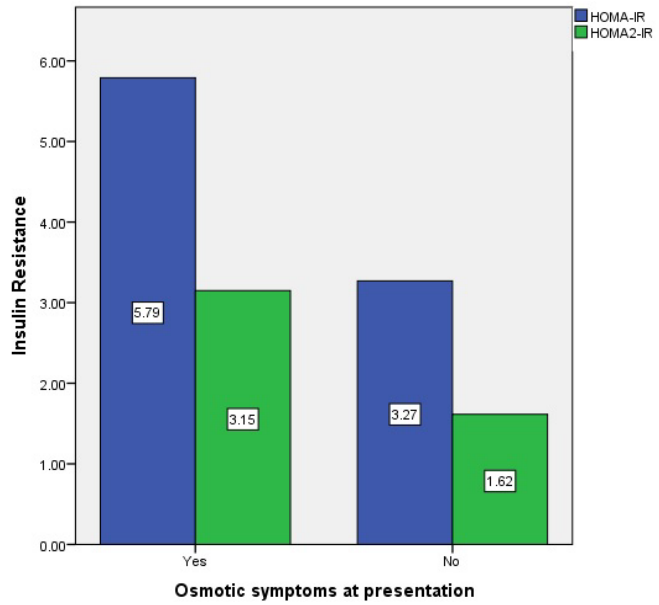

Figure 2: Bar diagram showing higher insulin resistance in cohort of patients with osmotic symptoms at presentation

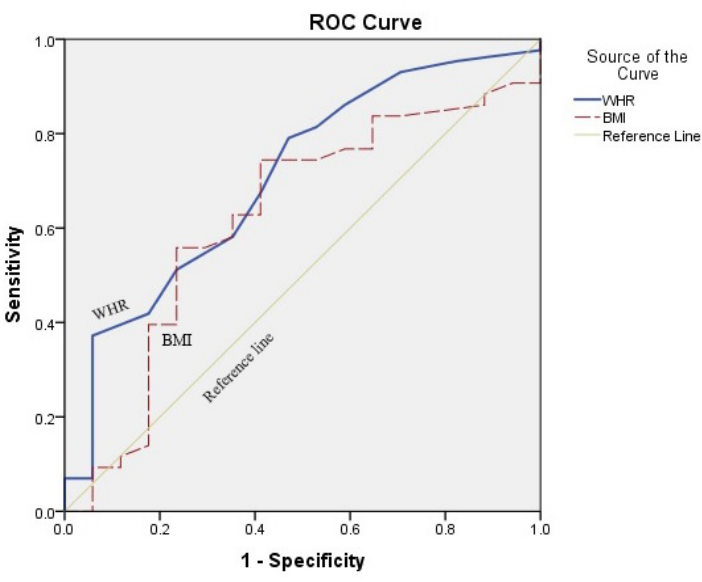

Figure 3: ROC (receiver operating characteristics) curve of BMI and WHR for prediction of patients with insulin resistance.

\begin{tabular}{|c|c|c|c|c|}
\hline & \multicolumn{2}{|l|}{ HOMA-IR } & \multicolumn{2}{|l|}{ HOMA2-IR } \\
\hline & Pearson's Correlation Coefficient & $p$-value & Pearson's Correlation Coefficient & $p$-value \\
\hline Age Group (years) & 0.015 & 0.908 & 0.002 & 0.988 \\
\hline Total Cholesterol (mmol/l) & 0.266 & $0.040^{*}$ & 0.237 & 0.069 \\
\hline Triglyceride (mmol/l) & 0.191 & 0.144 & 0.177 & 0.175 \\
\hline HDL Cholesterol (mmol/l) & 0.088 & 0.505 & 0.084 & 0.522 \\
\hline LDL Cholesterol (mmol/l) & 0.304 & $0.018^{*}$ & 0.264 & $0.041^{*}$ \\
\hline TC/HDL-C & 0.233 & 0.073 & 0.203 & 0.120 \\
\hline TG/HDL-C & 0.140 & 0.287 & 0.144 & 0.272 \\
\hline Body Mass Index (BMI) & 0.161 & 0.221 & 0.141 & 0.283 \\
\hline Waist Hip Ratio (WHR) & 0.284 & $0.028^{*}$ & 0.274 & $0.034^{*}$ \\
\hline BP systolic (mm Hg) & 0.496 & $<0.001^{*}$ & 0.406 & $<0.001^{*}$ \\
\hline BP diastolic (mm Hg) & 0.609 & $<0.001^{*}$ & 0.562 & $<0.001^{*}$ \\
\hline Family History & 0.449 & $<0.001^{*}$ & 0.335 & $0.009^{*}$ \\
\hline Dietary Habbit & 0.128 & 0.331 & 0.125 & 0.340 \\
\hline Dietary Frequency & 0.174 & 0.183 & 0.183 & 0.163 \\
\hline Exercise & 0.060 & 0.647 & 0.089 & 0.500 \\
\hline Vegetables intake & -0.104 & 0.428 & -0.159 & 0.226 \\
\hline Fruits intake & 0.071 & 0.591 & 0.038 & 0.772 \\
\hline Carbohydrates intake & 0.039 & 0.768 & 0.018 & 0.891 \\
\hline Oil intake & 0.255 & $0.049^{*}$ & 0.232 & 0.075 \\
\hline Salt intake & 0.050 & 0.707 & 0.005 & 0.971 \\
\hline Lifestyle & 0.683 & $<0.001^{*}$ & 0.661 & $<0.001^{*}$ \\
\hline Alcohol intake & 0.115 & 0.384 & 0.068 & 0.608 \\
\hline Smoking & 0.252 & 0.052 & 0.205 & 0.116 \\
\hline Tobacco & 0.249 & 0.055 & 0.257 & $0.048^{*}$ \\
\hline
\end{tabular}

*Statistically significant at $p<0.05$

Table 6: Correlation of insulin resistance with various cardiovascular risk factors. 
Citation: Basukala P, Jha B, Yadav BK, Shrestha PK (2018) Determination of Insulin Resistance and Beta-Cell Function Using Homeostatic Model Assessment in Type 2 Diabetic Patients at Diagnosis. J Diabetes Metab 9: 790. doi: 10.4172/2155-6156.1000790
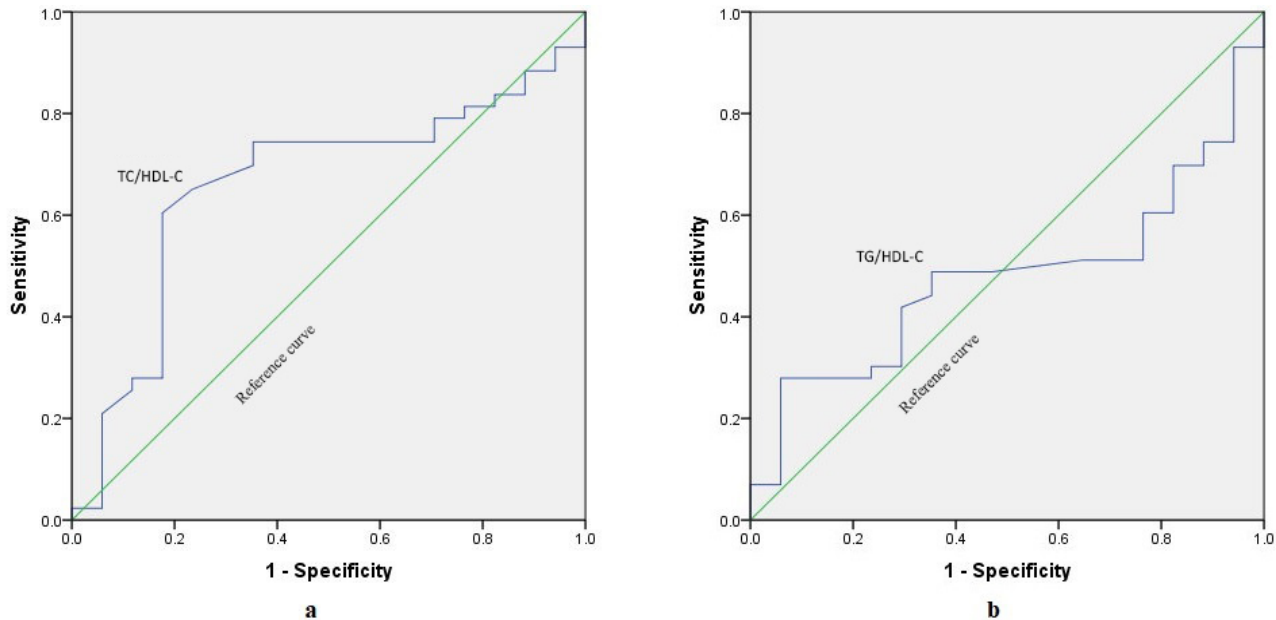

Figure 4: ROC (receiver operating characteristics) curve of a) TC/HDL-C; and b) TG/HDL-C ratio for detection of patients with insulin resistance.

\begin{tabular}{|c|c|c|c|c|}
\hline & \multicolumn{2}{|l|}{ HOMA \%B } & \multicolumn{2}{|l|}{ HOMA2 \%B } \\
\hline & Pearson's Correlation Coefficient & $p$-value & Pearson's Correlation Coefficient & $p$-value \\
\hline Age Group (years) & -0.196 & 0.133 & -0.208 & 0.110 \\
\hline Total Cholesterol (mmol/l) & -0.193 & 0.139 & -0.233 & 0.074 \\
\hline Triglyceride (mmol/l) & 0.085 & 0.517 & 0.063 & 0.630 \\
\hline HDL Cholesterol (mmol/l) & 0.000 & 0.999 & -0.008 & 0.954 \\
\hline LDL Cholesterol (mmol/l) & -0.259 & $0.046^{*}$ & -0.301 & $0.019^{*}$ \\
\hline TC/HDL-C & -0.185 & 0.156 & -0.223 & 0.087 \\
\hline TG/HDL-C & 0.134 & 0.309 & 0.117 & 0.372 \\
\hline Body Mass Index (BMI) & -0.030 & 0.818 & -0.058 & 0.659 \\
\hline Waist Hip Ratio (WHR) & -0.067 & 0.609 & -0.115 & 0.382 \\
\hline BP systolic $(\mathrm{mm} \mathrm{Hg})$ & -0.058 & 0.659 & -0.117 & 0.372 \\
\hline BP diastolic $(\mathrm{mm} \mathrm{Hg})$ & -0.005 & 0.972 & -0.066 & 0.615 \\
\hline Family History & -0.297 & $0.021^{*}$ & -0.342 & $0.007^{*}$ \\
\hline Dietary Habbit & -0.205 & 0.117 & -0.192 & 0.142 \\
\hline Dietary Frequency & 0.144 & 0.271 & 0.140 & 0.288 \\
\hline Exercise & 0.351 & $0.006^{*}$ & 0.309 & $0.016^{*}$ \\
\hline Vegetables intake & -0.224 & 0.086 & -0.193 & 0.139 \\
\hline Fruits intake & 0.079 & 0.547 & 0.071 & 0.591 \\
\hline Carbohydrates intake & -0.019 & 0.888 & -0.039 & 0.766 \\
\hline Oil intake & -0.348 & $0.006^{*}$ & -0.366 & $0.004^{*}$ \\
\hline Salt intake & -0.332 & $0.010^{*}$ & -0.307 & $0.017^{*}$ \\
\hline Lifestyle & -0.231 & 0.076 & -0.305 & $0.018^{*}$ \\
\hline Alcohol intake & -0.250 & 0.054 & -0.263 & $0.042^{*}$ \\
\hline Smoking & -0.111 & 0.397 & -0.134 & 0.309 \\
\hline Tobacco & -0.066 & 0.616 & -0.090 & 0.496 \\
\hline
\end{tabular}

*Statistically significant at $p<0.05$

Table 7: Correlation of beta-cell function indices with anthropometric/metabolic variables.

\begin{tabular}{|c|c|c|c|c|}
\hline Cardiovascular Risk Factors & Areas under the ROC curve & $95 \%$ Confidence interval & $p$-value & Optimal cut off for predicting IR \\
\hline BMI & 0.622 & $0.459-0.784$ & 0.144 (NS) \\
\hline WHR & 0.705 & $0.559-0.851$ & 27.70 \\
\hline
\end{tabular}

*Statistically significant at $p \leq 0.05$;

NS: Non-significant

Table 8: Comparison of BMI and WHR using areas under ROC (receiver operating characteristic) curve for prediction of insulin resistance (HOMA2-IR).

\begin{tabular}{|c|c|c|c|c|}
\hline Serum lipoprotein ratios & Area under the ROC curve & $\mathbf{9 5 \%}$ Confidence interval & $\boldsymbol{p}$-value & Optimal cutoff for predicting IR \\
\hline TC/HDL-C ratio & 0.70 & $0.509-0.812$ & $0.05^{*}$ & 4.47 \\
\hline TG/HDL-C ratio & 0.50 & $0.329-0.625$ & 0.78 (NS) \\
\hline
\end{tabular}

*Statistically significant at $p \leq 0.05$;

NS: Non-significant

Table 9: Serum lipoprotein ratios and the areas under ROC (receiver operating characteristic) curve for detection of insulin resistance (HOMA2-IR). 
screening ability for insulin resistance ( $\mathrm{AUC}=0.7 ; \mathrm{P}=0.05$ ) with an optimal cutoff of 4.47 whereas TG/HDL-C ratio failed as a screening tool for insulin resistance as the AUC obtained for it was unacceptable rendering its optimal cutoff indefinable (Table 8).

\section{Discussion}

This study demonstrated the magnitude of IR and BCF and their associated metabolic risk factors among the 60 new T2DM attendees of an out-patient department of a tertiary care hospital in Nepal. The mean fasting serum glucose of these recently diagnosed diabetic patients was high $(10.68 \pm 3.46 \mathrm{mmol} / \mathrm{l})$ and the mean fasting C-peptide was found to be on the upper normal range $(875.41 \pm 294.53 \mathrm{pmol} / \mathrm{l})$. It has been stated that in early diabetes, glucose regulation is of secondary importance to maintenance of basal insulin secretion. So the basal plasma glucose rises in an attempt to stimulate the reduced numbers of beta cells to secrete normal basal insulin. This forces the remaining beta cells to operate nearer their maximal capacity [17].

Both HOMA1-IR and HOMA2-IR in this study showed significant correlations with WHR, while the correlation between BMI and HOMA1-IR or HOMA2-IR was poor. Mishra et al. from India [18] also found strong correlation of HOMA1-IR with WHR. A similar result was obtained in a large study from Europe [19]. In the Atherosclerosis Risk in Community study [20] also, the AUC of WHR for prediction of IR and T2DM was significantly greater than that of BMI. All these studies have shown that WHR is a good predictor of IR and can thus be used to identify at risk individuals. Although BMI may be a simple noninvasive method for assessing obesity and excessive fat stores, it is less reliable in comparing body composition [21]. One of the reasons for reduced reliability of $\mathrm{BMI}$ is that there may be identical distributions of BMI in two populations but they could still reflect large differences with respect to the accumulation of intra-abdominal fat responsible for development of insulin resistance. The findings in this study indicated $\mathrm{BMI}$ to be an unreliable measure for associating insulin resistance and T2DM (Table 3). Instead, WHR is a more reliable marker of insulin resistance.

This study also demonstrated the more reduced insulin sensitivity (HOMA \%S) compared to reduction in beta cell function (HOMA\% B) in new T2DM patients (Table 9). This can be explained by the fact that South Asians have preferential fat deposition in the abdominal region which is associated with reduced insulin-mediated glucose disposal [22]. Thus South Asian population being more prone to abdominal obesity and also low muscle mass, have been hypothesized to develop insulin resistance and consequently type 2 diabetes sooner than the population of European descent [21]. In addition, higher prevalence of low birth weight (indicator of intrauterine malnutrition) along with low muscle mass in their lifetime has also been attributed for the higher prevalence of diabetes in these populations [23]. Poor nutrition in foetal life and infancy predisposes to fewer beta cells and stimulates the development of thrifty genes that confers a survival advantage in such nutrient deficient environments. But it becomes disadvantageous in later life when nutrition becomes abundant and increased demand for insulin outstrips the capacity of production, predisposing the individual to development of T2DM $[24,25]$.

A positive family history for DM is a controversial contributing risk factor for developing IR and DM. Some researchers have demonstrated a significant association between a positive family history of DM and IR [26], whereas other studies strongly disagree [27]. Subjects having positive family history of T2DM are found to have low insulin like growth factor binding protein-1 levels which is associated with adolescent obesity and impaired beta-cell compensation relative to lower insulin sensitivity [26]. Regarding stressful lifestyle as one of the causative factors for development of insulin resistance and subsequent cardiovascular risks, a study done by Räikkönen et al. [28] shows a close correlation of stress related factors and insulin resistance whereas in a study done by Zareian et al. [29], there was no significant correlation between insulin resistance and stress score. This study didn't use any type of questionnaire (eg. Osipow questionnaire as used by Zareian et al.) to assess the level of stress. The role of stress in development of insulin resistance has been associated with accumulation of intraabdominal fat, hyperlipidaemia, hypertension, altered insulin/ glucose homeostasis, and an increased level of plasminogen activator inhibitor-1 [28].

Concerning daily oil intake, the type of fatty acids consumed is more important than total fat in the diet. Long-term intake of saturated and trans fatty acids has been associated with insulin resistance [30] Frequent tobacco consumers have also been found to develop early insulin resistance syndrome (IRS). Nicotine and cigarette smoking have been recognized to cause the level of stress hormone, cortisol, to rise in excess to induce insulin resistance [31]. Regarding vegetable intake, the pathway through which vegetables influence health is not well understood. However, dietary fiber in vegetables has been known to limit insulin response following meals by slowing macronutrient absorption through delayed gastric emptying [32].

Osmotic symptoms of hyperglycemia are the most strongly endorsed symptoms in DM. Symptoms of thirst; dry mouth, higher affinity for sweets; polyuria and frequency of night urination appear to be specific for hyperglycaemia in DM. Although osmotic symptoms may not appear in the early stages of impaired fasting glucose, it emerges with more prolonged or severe hyperglycaemia during the later course of the disease when islet cell damage and insulin resistance has heightened their peak [33]. Thus, in such patients, presence of osmotic symptoms can be associated with higher degree of insulin resistance.

Regarding lipid parameters, LDL cholesterol was found to be significantly correlated with HOMA-IR and HOMA \%B values. Diabetes is well known for characteristic dyslipidaemia which includes elevated TG, low HDL-C, and preponderance of small dense LDL particles. Any relation between LDL particles and insulin resistance could probably be exerted through the effect of insulin on lipoprotein metabolism [34]. Accumulation of excess cholesterol in beta-cells causes lipotoxicity by decreasing expression of transcription factors required for beta-cell development and survival [35]. Roehrich et al. [36] have reported LDL-C above $6 \mathrm{mmol} / \mathrm{l}$ to induce apoptosis of betacells. Moderate exercise has been found to improve insulin sensitivity by reducing visceral and abdominal subcutaneous fat and maintains betacell function by decreasing lipotoxicity to beta-cells [37]. As for alcohol consumption, moderate alcohol intake has been associated with lower risk of T2DM whereas high and chronic intake leads to abdominal obesity and accelerates pancreatic fibrosis and beta-cell damage [38].

This study indicated TC/HDL-C ratio to be used as surrogate marker for insulin resistance and not TG/HDL-C ratio. TG/HDL-C ratio has been demonstrated to fail to predict insulin resistance in people of African-American ethnicity by Anne et al. [39]. However in a study done by Ray et al. [10] both TC/HDL-C ratio and TG/HDL-C ratios were found to be significantly correlated with HOMA-IR with excellent AUC value of 0.8 for TG/HDL-C ratio and acceptable AUC value of 0.78 for TC/HDL-C ratio. Thus, it is important to note that, although TG/HDL-C ratio has been recommended as surrogate for insulin resistance, the relation between TG and TG/HDL-C with 
Citation: Basukala P, Jha B, Yadav BK, Shrestha PK (2018) Determination of Insulin Resistance and Beta-Cell Function Using Homeostatic Model Assessment in Type 2 Diabetic Patients at Diagnosis. J Diabetes Metab 9: 790. doi: 10.4172/2155-6156.1000790

insulin may differ by ethnicity and hence in certain populations, using it to determine insulin resistance may not be appropriate [40,41].

\section{Conclusion}

Our study found more reduced insulin sensitivity compared to reduction in beta-cell function in new T2DM patients. Also, the degree of insulin resistance in newly diagnosed diabetic patients who have not yet developed osmotic symptoms was lesser than those who have. These findings suggest that early intensive treatment addressing insulin resistance is, in fact, the best approach to slow the development of the disease and its progression, and to possibly retard the development of chronic complications of T2DM. This necessitates the use of easy and reliable diagnostic tools such as the HOMA model for early detection of insulin resistance and beta-cell failure. Although simple, the application of HOMA model using C-peptide might be constrained in resource limited country like ours. Under such scenario, the use of cheaper and reliable surrogates of insulin resistance like the WHR and TC/HDL ratio as verified by this study should be effectively used.

\section{Authors' Contributions}

PB carried out the study, analysed the data and prepared the manuscript. PKS designed the study and supervised the work. BJ supervised the work. All authors have read and approved the manuscript.

\section{Acknowledgements}

We thank the staffs of Biochemistry Department, Medicine Department, and Diabetes Diet Plan and Counselling Department, TUTH, for helping us in carrying out this study.

\section{Competing Interests}

No competing interests.

\section{Ethics Approval and Consent to Participate}

Written permission to conduct the study was obtained from Institutional Review Board, Research Department, Institute of Medicine. Informed written consent was explained and obtained from the all participants.

\section{Funding}

This study was funded by University Grants Commission (UGC), Sanothimi Bhaktapur.

\section{References}

1. Antuna-Puente B, Disse E, Rabasa-Lhoret R, Laville M, Capeau J, et al. (2011) How can we measure insulin sensitivity/resistance? Diabetes Metab 37: $179-188$.

2. Saisho Y (2014) Postprandial C-peptide Index: The Best Marker of Beta Cell Function? Int J Diab Clin Diagn.

3. DeFronzo RA, Tobin JD, Andres R (1979) Glucose clamp technique: a method for quantifying insulin secretion and resistance. Am J Physiol 237: G214-G223.

4. Matthews D, Hosker J, Rudenski A, Naylor B, Treacher D, et al. (1985) Homeostasis model assessment: insulin resistance and $\beta$-cell function from fasting plasma glucose and insulin concentrations in man. Diabetologia 28 : 412-419

5. Levy JC, Matthews DR, Hermans MP (1998) Correct homeostasis mode assessment (HOMA) evaluation uses the computer program. Diabetes care 21: 2191-2192.

6. https://wwwdtuoxacuk/homacalculator/

7. Wallace TM, Levy JC, Matthews DR (2004) Use and abuse of HOMA modeling. Diabetes care 27: 1487-1495

8. Li X, Zhou Z, Qi H, Chen X, Huang G (2004) [Replacement of insulin by fasting C-peptide in modified homeostasis model assessment to evaluate insulin resistance and islet beta cell function]. Zhong nan da xue xue bao Yi xue ban 29: 419-423.

9. Rajala U, Laakso M, Päivänsalo M, Pelkonen O, Suramo I, et al. (2002)
Low insulin sensitivity measured by both quantitative insulin sensitivity check index and homeostasis model assessment method as a risk factor of increased intima-media thickness of the carotid artery. J Clin Endocrin Metab 87: 5092-5097.

10. Ray S, Bairagi AK, Guha S, Ganguly S, Ray D, et al. (2012) A simple way to identify insulin resistance in non-diabetic acute coronary syndrome patients with impaired fasting glucose. Indian J Endocrinol Metab 16: 460-464.

11. Kinosian B, Glick H, Preiss L, Puder KL (1995) Cholesterol and coronary heart disease: predicting risks in men by changes in levels and ratios. J Investig Med 43: 443-450.

12. Rajappa M, Sridhar M, Balachander J, Sethuraman K, Rajendiran KS (2014) Lipoprotein ratios as surrogate markers for insulin resistance in South Indians with normoglycemic nondiabetic acute coronary syndrome. ISRN Endocrinol 2014: 981524

13. Ray S, Talukdar A, Sonthalia N, Saha M, Kundu S, et al. (2015) Serum lipoprotein ratios as markers of insulin resistance: A study among non-diabetic acute coronary syndrome patients with impaired fasting glucose. Indian J Med Res 141: 62-67.

14. Tandon YSMGN, Marwaha RK (2013) A study of insulin resistance by HOMA-IR and its cut-off value to identify metabolic syndrome in urban Indian adolescents. $\mathrm{J}$ Clin Res Pediatr Endocrinol 5: 245-251.

15. Kalaichelvi S, Somasundram K (2016) Prevalence of insulin resistance among patients with cirrhosis of liver in Government Royapettah Hospital, Chennai. IAIM 3: 21-27

16. Hosmer DW, Lemeshow S (2000) Multiple logistic regression, Wiley Online Library.

17. Turner RC, Holman RR, Hockaday TDR (1976) Beta Cell Deficiency in Maturity Onset Diabetes. Clin Sci 51: 18

18. Misra A, Vikram N, Arya S, Pandey R, Dhingra V, et al. (2004) High prevalence of insulin resistance in postpubertal Asian Indian children is associated with adverse truncal body fat patterning, abdominal adiposity and excess body fat Int J Obes Relat Metab Disord 28: 1217-1226.

19. Kondaki K, Grammatikaki E, Pavón DJ, Manios Y, González-Gross M, et al (2011) Comparison of several anthropometric indices with insulin resistance proxy measures among European adolescents: The Helena Study. Eur J Pediatr 170: 731-739.

20. Stevens J, Couper D, Pankow J, Folsom AR, Duncan BB, et al. (2001) Sensitivity and specificity of anthropometrics for the prediction of diabetes in a biracial cohort. Obes Res 9: 696-705.

21. Group D-DS (2003) Age, body mass index and type 2 diabetes-associations modified by ethnicity. Diabetologia 46: 1063-1070.

22. Després JP, Nadeau A, Tremblay A, Ferland M, Moorjani S, et al. (1989) Role of deep abdominal fat in the association between regional adipose tissue distribution and glucose tolerance in obese women. Diabetes 38: 304-309.

23. Wang J, Thornton JC, Russell M, Burastero S, Heymsfield S, et al. (1994) Asians have lower body mass index (BMI) but higher percent body fat than do whites: comparisons of anthropometric measurements. Am J Clin Nutr 60 : 23-28.

24. Hales C, Barker D, Clark P, Cox L, Fall C, et al. (1991) Fetal and infant growth and impaired glucose tolerance at age 64. BMJ 303: 1019-1022.

25. Barker DJ, Hales CN, Fall C, Osmond C, Phipps K, et al. (1993) Type 2 (non-insulin-dependent) diabetes mellitus, hypertension and hyperlipidaemia (syndrome X): relation to reduced fetal growth. Diabetologia 36: 62-67.

26. Arslanian SA, Bacha F, Saad R, Gungor N (2005) Family history of type 2 diabetes is associated with decreased insulin sensitivity and an impaired balance between insulin sensitivity and insulin secretion in white youth Diabetes care 28: 115-119.

27. Goldfine AB, Bouche C, Parker RA, Kim C, Kerivan A, et al. (2003) Insulin resistance is a poor predictor of type 2 diabetes in individuals with no family history of disease. Proc Natl Acad Sci USA 100: 2724-2729.

28. Räikkönen K, Keltikangas-Järvinen L, Adlercreutz H, Hautanen A (1996) Psychosocial stress and the insulin resistance syndrome. Metabolism 45: 1533-1538.

29. Zareian P, Ghasemi F, Abtahi SR, Behzadi S (2016) A Comparison Between Job Stress and Insulin Resistance Among the Hospital Medical Staff. J Arch 
Citation: Basukala P, Jha B, Yadav BK, Shrestha PK (2018) Determination of Insulin Resistance and Beta-Cell Function Using Homeostatic Model Assessment in Type 2 Diabetic Patients at Diagnosis. J Diabetes Metab 9: 790. doi: 10.4172/2155-6156.1000790

Page 11 of 11

Mil Med.

30. Riccardi G, Giacco R, Rivellese A (2004) Dietary fat, insulin sensitivity and the metabolic syndrome. Clin Nutr 23: 447-456.

31. Ambrose JA, Barua RS (2004) The pathophysiology of cigarette smoking and cardiovascular disease: an update. J Am Coll Cardiol 43: 1731-1737.

32. Ray TK, Mansell KM, Knight L, Malmud L, Owen O, et al. (1983) Long-term effects of dietary fiber on glucose tolerance and gastric emptying in noninsulindependent diabetic patients. Am J Clin Nutr 37: 376-381.

33. Warren RE, Deary IJ, Frier BM (2003) The symptoms of hyperglycaemia in people with insulin-treated diabetes: classification using principal components analysis. Diabetes Metab Res Rev 19: 408-414.

34. Reaven GM, Chen YD, Jeppesen J, Maheux P, Krauss RM (1993) Insulin resistance and hyperinsulinemia in individuals with small, dense low density lipoprotein particles. J Clin Invest 92: 141.

35. Ishikawa M, Iwasaki Y, Yatoh S, Kato T, Kumadaki S, et al. (2008) Cholesterol accumulation and diabetes in pancreatic $\beta$-cell-specific SREBP-2 transgenic mice: a new model for lipotoxicity. J Lipid Res 49: 2524-2534.

36. Roehrich ME, Mooser V, Lenain V, Herz J, Nimpf J, et al. (2003) Insulin- secreting $\beta$-cell dysfunction induced by human lipoproteins. J Biol Chem 278 : 18368-18375

37. Slentz CA, Tanner CJ, Bateman LA, Durheim MT, Huffman KM, et al. (2009) Effects of exercise training intensity on pancreatic $\beta$-cell function. Diabetes care 32: 1807-1811.

38. Yue F, Zhang X, Zhang H, Jiang X, Gao L, et al. (2012) Association of alcohol consumption with the impaired $\beta$-cell function independent of body mass index among Chinese men. Endocr J 59: 425-433.

39. Sumner AE, Harman JL, Buxbaum SG, Miller III BV, Tambay AV, et al. (2010) The triglyceride/high-density lipoprotein cholesterol ratio fails to predict insulin resistance in African-American women: an analysis of Jackson Heart Study. Metab Syndr Relat Disord 8: 511-514.

40. Bovet P, Faeh D, Gabriel A, Tappy L (2006) The prediction of insulin resistance with serum triglyceride and high-density lipoprotein cholesterol levels in an East African population. Arch Intern Med 166: 1236-1237.

41. Ford ES, Li C, Imperatore G, Cook S (2006) Age, Sex, and Ethnic Variations in Serum Insulin Concentrations Among US Youth Findings from the National Health and Nutrition Examination Survey 1999-2002. Diabetes Care 29: 26052611. 